\title{
Toxicity Studies of Photo-Oxidized Vegetable Oil and Water Underlying Crude Oil in Albino Rats
}

\author{
Ibiba F Oruambo ${ }^{*}$, G A Obediah ${ }^{1}$ and G K Fekarurhobo ${ }^{2}$ \\ ${ }^{1}$ Department of Biochemistry, Rivers State University \\ ${ }^{2}$ Department of Chemistry, Rivers State University
}

Received: January 11, 2018; Accepted: March 27, 2018; Published: March 30, 2018

*Corresponding author: Ibiba F Oruambo, Department of Chemistry, Biochemistry, Rivers State University of Science and Technology Nkpolu-Port Harcourt, Rivers State, Nigeria; E-mail: ibibaforuambo@yahoo.com

\begin{abstract}
Prolonged exposure to sunlight or florescent light of vegetable oils contained in transparent vessels are susceptible to photo-induced oxidation that results in formation of potentially toxic peroxides, alcohols, ketones, aldehydes and carboxylic acid. Similarly, crude oil spillage land and water pose severe health risks to people who are chronically exposed when photo-oxidation takes place, especially shallow water subsistent fishermen and farmers. Hence, the main objective of the study is to ascertain probable toxicities of photo-oxidized vegetable oil and spilled crude oil to people who chronically consume or are exposed to them. This was achieved by determining whether or not treatment of albino rats with photo-exposed vegetable oil or water underlying spilled crude oil would adversely alter parameters of organ function markers [OFMs] as indicators of probable organ toxicity. Samples of virgin olive oil were exposed in transparent glass bottles to fluorescent lighting continuously for 336 hours. Control samples were not exposed. Intermittently, aliquots of exposed samples were subjected to peroxide value analysis. A sample of crude oil was suspended in water in a glass container and exposed to sunlight continuously for 336 hours. Control sample was keep in a dark cupboard. Similarly, both sample types were analyzed by GC-MS. Thirty-six [36] male albino rats were divided into nine groups of four animals each. All treated albino rats were given olive oil or water underlying crude oil mixed with rat feed in a ratio of 2:10 or 4:10 v/w respectively and given drinking water ad libitum except the external control. The treatment was daily for 6 weeks, at the end of which rats were sacrificed by decapitation following manual stunning. Blood sample of approximately $5 \mathrm{ml}$ was collected from each rat for liver and kidney function indicators [OFMs] analysis. The results revealed increasing peroxide values of fluorescent lighting-exposed virgin oil as a function of increasing duration [in hours], from zero hour [control] of $2 \mathrm{meq} / \mathrm{kg}$ to $56 \mathrm{meq} / \mathrm{kg}$ at 336hours of exposure, and four different levels of effect on liver and kidney function indicators [OFMs]. In conclusion, the continuous exposure of vegetable oil [in transparent container] and water underlying crude oil to indoor fluorescent lighting or to atmospheric sunlight respectively result in the oxidation of the polycyclic aromatic hydrocarbons to potentially toxic secondary metabolites which result to the probable hepato-and nephrotoxicities by treatment of adult male albino rats as judged by effects to liver and kidney Function Indices. The obvious implication of these results is that vegetable oil should be stored in non-transparent/opaque bottles protected from fluorescent lighting while on supermarket shelves and in open general markets. Commercial producers should be mandated by law/legislation to implement this.
\end{abstract}

\section{Introduction}

Adverse health effects, such as heart disease [arteriosclerosis - formation of arterial plaques], kidney and liver dysfunction may occur as a result of the chronic consumption of poly-saturated fatty acids as contained in animal meat. Consequently, nutritionists and medical doctors have recommended the consumption of poly-unsaturated fatty acids derived from plants sources, such as vegetable oil. Poly-unsaturated fatty acids, among other properties, have significantly low melting points that are below physiological in vivo temperature of 37 degrees centigrade, and therefore do not cause arterial plaques.

However, vegetable oils are traditionally and conventionally stored/packaged in transparent containers which are exposed to sunlight and florescent lighting for hours on end in the general market and superstores, respectively. This is the problem: prolonged exposure to sunlight or florescent light of vegetable oils contained in transparent vessels are susceptible to photo-induced oxidation that results in formation of potentially toxic peroxides, alcohols, ketones, aldehydes and carboxylic acid. Similarly, crude oil spillage land and water pose severe health risks to people who are chronically exposed when photo-oxidation takes place, especially shallow water subsistent fishermen and farmers. The following questions arise from the widespread consumption of photo-exposed vegetable oil, and occupational exposure to photoexposed crude oil spills regarding the inherent and probable adverse health implications:

1. Does photo-exposure of vegetable oil and crude oil spill result in oxidation and formation of potentially toxic products such as peroxides and PAH-epoxides?

2. Are these products actually toxic to the liver and kidney in the albino rat under controlled laboratory conditions as judged by effects to Organ Function Markers [OFMs]? 
It has been established that no significant degree of degradation of crude oil can occur without the predisposition of its components to microbial susceptibility by their preliminary atmospheric oxidation [3]. The reactions convert the hydrocarbon components in crude oil to their oxygenated analogues, which are reactive enzyme substrates. The oxidation reactions can be induced thermally and photo-chemically, although the latter [being faster in their reactions rates] are thought to predominate [4]. The mechanism of reactions can either be free-radical or concerted but the primary products are usually peroxides which are known to readily decompose thermally or photo-chemically or by rearrangement to give other compounds; the peroxy bond being one of the weakest in the organic compounds [1,8]. The secondary products of these decompositions are aldehydes, ketones, epoxides, alcohols and carboxylic acids which commonly constitute components of the water underlying a spillage after one day [2]. The toxicity of these products implies that damage to the water body may have been done before "prompt action" is taken to remove the spilled crude oil, the only mitigating factor being the volume of the water relative to that of the spillage .

In the oxidation reactions, the components with chromophores are more susceptible. Thus, the alkenes and aromatic compounds which are unsaturated are more reactive than the alkanes which are saturated. Unfortunately, the oxidation of the more reactive components renders them more toxic. The benzo-[a]-pyrene epoxide, derived by oxidation of benzo-[a]-pyrene is several times more toxic than its precursor [Shigeyuki et al, 2000; Wessel et al, 2010]. This is also true of the hydrocarbons oxidation products. Their toxicity derives from their power to bind to proteins, nucleotides, enzymes, nucleic acids, hemoglobin and other cell components. The presence of some of the aromatic components [e.g. benzopyrene] in crude oil can render it more photooxidizable by their ability to sensitize the formation of singlet oxygen [102], which is more reactive than ground state triplet [302]. Thus, adsorption and absorption of such components of crude oil onto and into the skin and exposure to sunlight can result in the formation of 102 epi - or endo-dermally. 102 in living cells can lead to the formation of reactive oxygen species [ROS] which can kill the cells if their defensive mechanisms are overwhelmed [10]. Toxicity of crude oil was shown to induce abnormal changes in the blood constituents of adult albino rats following dermal exposure to Bonny light crude oil [5]. In addition, Bonny light crude oil has also been shown to cause a dose related decrease in activities of the liver function markers Amino Transferases [SGOT and SGPT] in Guinea-Pigs treated by skin application [7]. The same photo-oxidation mechanism applies to vegetable oil consisting primarily of poly-unsaturated hydrocarbons in the form of fatty acids; the primary products are peroxides while secondary decomposition products are aldehydes, ketones, carboxylic acid and other oxygenated species.

The main objective of the study is to ascertain the probable toxicities of photo-oxidized vegetable oil and water underlying crude oil by determining whether or not extended treatment of male albino rats with photo-exposed vegetable oil or water underlying crude oil would adversely alter parameters of liver and kidney organ function markers [OFMs] as indicators of probable organ toxicity.

\section{Materials and Methods \\ Indoor Exposure of Virgin Olive Oil}

Samples of virgin olive oil were exposed in transparent glass bottles to fluorescent lighting continuously for 336 hours. Control samples were not exposed. Intermittently, aliquots of exposed samples were subjected to peroxide value analysis. Both sample types were analyzed by GC-MS, as well.

\section{Outdoor Atmospheric Exposure of Water-Suspended Crude Oil}

A sample of crude oil was suspended in water in a glass container and exposed to sunlight continuously for 336 hours. Control sample was keep in a dark cupboard. Similarly, both sample types were analyzed by GC-MS.

\section{Peroxide Value Test}

This was done following a standard procedure outlined in AOAC official method Cd 8-53 [AOAC, 1993]

\section{Animal Treatment}

Male albino rats were divided into nine groups of four animals each and treated as follows:

\begin{tabular}{|c|c|}
\hline Group & Treatment \\
\hline A & $\begin{array}{l}2 \mathrm{ml} \text { of fluorescent lighting exposed virgin } \\
\text { olive oil (characterized as photo-oxidized } \\
\text { virgin olive oil, FPVOO) }\end{array}$ \\
\hline B & $\begin{array}{l}4 \mathrm{ml} \text { of fluorescent lighting exposed virgin } \\
\text { olive oil (characterized as photo-oxidized v } \\
\text { irgin olive oil, FPVOO) }\end{array}$ \\
\hline $\mathrm{C}$ & $\begin{array}{l}2 \mathrm{ml} \text { of sunlight exposed virgin olive oil } \\
\text { (characterized as atmospherically photo- } \\
\text { oxidized virgin olive oil, APVOO) }\end{array}$ \\
\hline $\mathrm{D}$ & $\begin{array}{l}4 \mathrm{ml} \text { of sunlight exposed virgin olive oil } \\
\text { (characterized as atmospherically photo- } \\
\text { oxidized virgin olive oil, APVOO) }\end{array}$ \\
\hline $\mathrm{E}$ & $\begin{array}{l}2 \mathrm{ml} \text { non-exposed virgin olive oil (i.e. control, } \\
\text { characterized simply as virgin olive oil, VOO) }\end{array}$ \\
\hline $\mathrm{F}$ & $\begin{array}{l}4 \mathrm{ml} \text { non-exposed virgin olive oil (i.e. control, } \\
\text { characterized simply as virgin olive oil, VOO) }\end{array}$ \\
\hline G & $\begin{array}{l}2 \mathrm{ml} \text { sunlight-exposed-water-underlyingcrude } \\
\text { oil (characterized as photo-oxidized crude oil, } \\
\text { PCO) }\end{array}$ \\
\hline $\mathrm{H}$ & $\begin{array}{l}4 \mathrm{ml} \text { sunlight-exposed-water-underlyingcrude } \\
\text { oil (characterized as photo-oxidized crude oil, } \\
\text { PCO) }\end{array}$ \\
\hline
\end{tabular}


I Drinking water only (i.e. external control)

All treated albino rats were given olive oil or water underlying crude oil mixed with rat feed in a ratio of $2: 10$ or $4: 10 \mathrm{v} / \mathrm{w}$ respectively and given drinking water ad libitum.

The treatment was daily for 6 weeks, at the end of which rats were sacrificed by decapitation following manual stunning

\section{Blood collection and processing for analysis of liver and kidney function markers [OFMs]}

Blood sample of approximately $5 \mathrm{ml}$ was collected from each rat in heparin sample bottles immediately after decapitation. These bottles were transferred into a container containing ice and were covered immediately to avoid exposure to ambient light and heat. The plasma was separated from the blood samples by centrifugation at $1000 \mathrm{rpm}$ for 30 minutes with an RP-1000 Centrifuge. The plasma was stored in firmly capped vials at $-20^{\circ} \mathrm{C}$ until ready for analysis.

Preparation of virgin vegetable oil and water underlying crude oil for PAHs and TPH assay by GC-MS

The method of sample preparation of photo-oxidized and non-oxidized virgin oil, non-exposed and sunlight exposed water underlying crude oil for PAHs and TPH assay was adopted from Obediah and Oruambo [6]. Liquid-liquid extraction of the virgin oil and water underlying crude oil was carried out using dichloromethane [analytical grade]. Prior to use, all glassware was thoroughly washed and dried overnight in an oven at high temperature. At the time of use, the funnel was washed vigorously for several minutes with dichloromethane and allowed to drain to complete dryness in a fume cupboard. $3 \mathrm{ml}$ of the virgin oil or water underlying crude oil was added to $5 \mathrm{ml}$ of dichloromethane and the mixture was vigorously shaken for $5 \mathrm{~min}$ and allowed to settle and separate. 10 min later the organic stratum was collected and the process was repeated with the aqueous layer twice. The three portions of the organic phase were pooled and evaporated to $2 \mathrm{ml}$ volume using a rotary evaporator.

\section{Organ Function Markers [OFMs]}

The following OFMs were measured in the plasma samples using Randox kits:

1. Liver: serum glutamate oxaloacetate transaminase [SGOT], serum glutamate pyruvate transaminase [SGPT], total and direct bilirubin, gamma glutamyl transferase [GGT], total protein, Albumin, Alkaline Phosphatase.

2. Kidney: Blood Urea Nitrogen [BUN], Electrolytes, and Creatinine were measured in all serum/plasma samples by standard procedures.

\section{Results}

\section{Discussion}

The premise of this study suggests that chronic exposure of virgin vegetable [olive] oil or water-suspended bonny light crude oil to either fluorescent lighting or sunlight [atmospheric] would result in photo-induced oxidation of the polycyclic aromatic hydrocarbons contents. The chromatograms in Figures 3 and 4 clearly demonstrate this event and therefore agree with the premise. The total amount of PAH reduced from 18,080.627ppm in non-exposed [control] crude oil to $1,026.828 \mathrm{ppm}$ [or $94 \%$ decrease], in atmospherically-exposed water-suspended crude oil, suggesting a photo-enabled degradation of the PAHs. Also the peroxide value result demonstrate that photo-oxidation took place in the virgin oil exposed to fluorescent light.

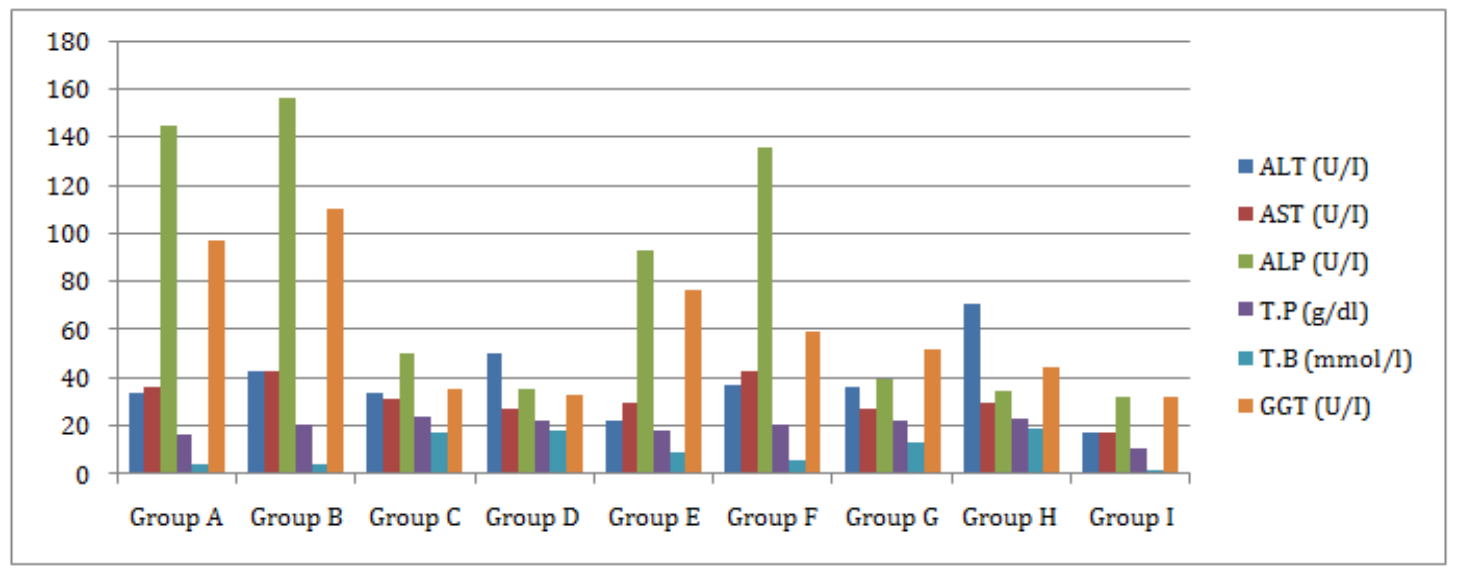

Figure 1: Liver function levels in serum of rats treated with fluorescent lighting exposed and sunlight exposed virgin oil and water underlying crude oil.

In Figure 3, the peaks of the 16 PAHs of the non-exposed crude oil [i.e. control] are prominent showing that the PAHs were not oxidized. On the other hand, in Figure 4, the peaks in the chromatogram are of the PAHs contained in the sunlight-exposed water underlying crude oil shown to be stratified and grossly stunted, suggesting that the PAHs were oxidized, therefore the resultant total hydrocarbons were captured in the chromatogram as TPH. 


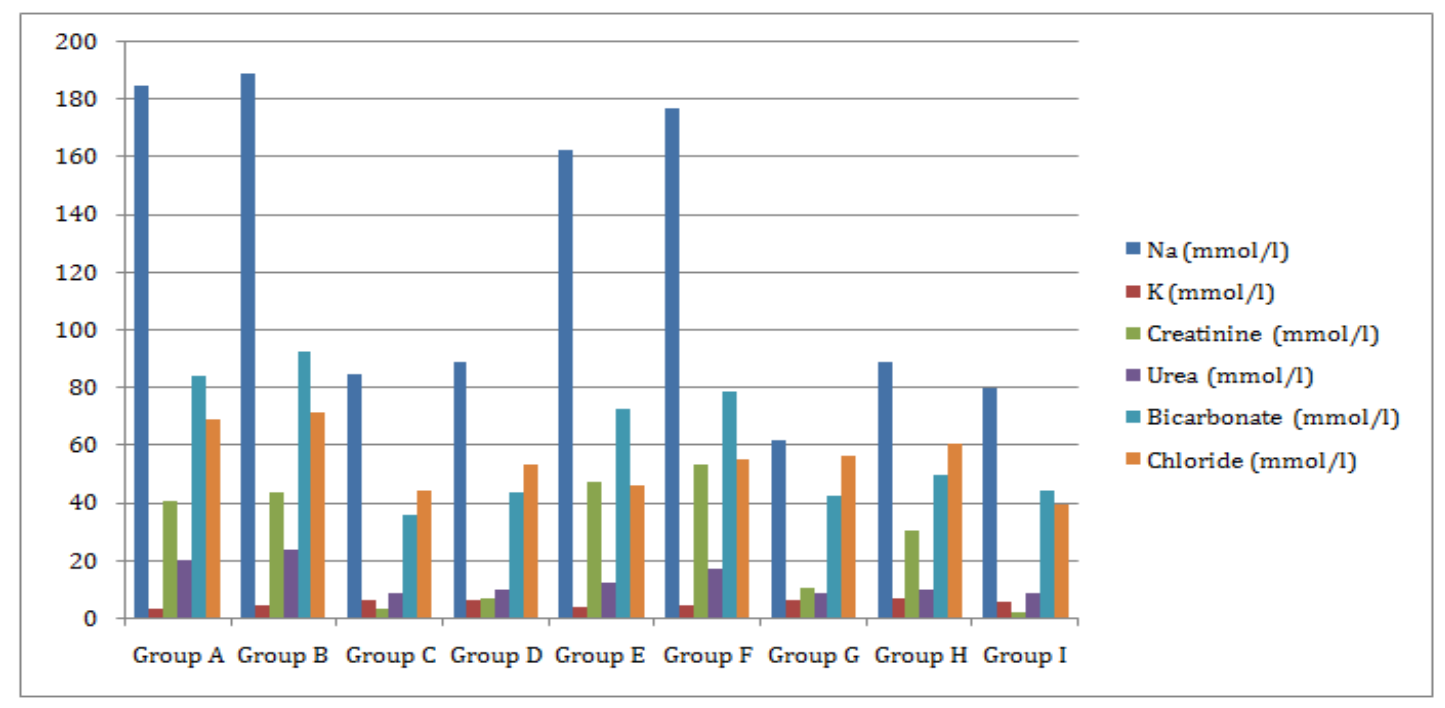

Figure 2: Kidney function levels in serum of rats treated with fluorescent lighting exposed and sunlight exposed virgin oil and water underlying crude oil.

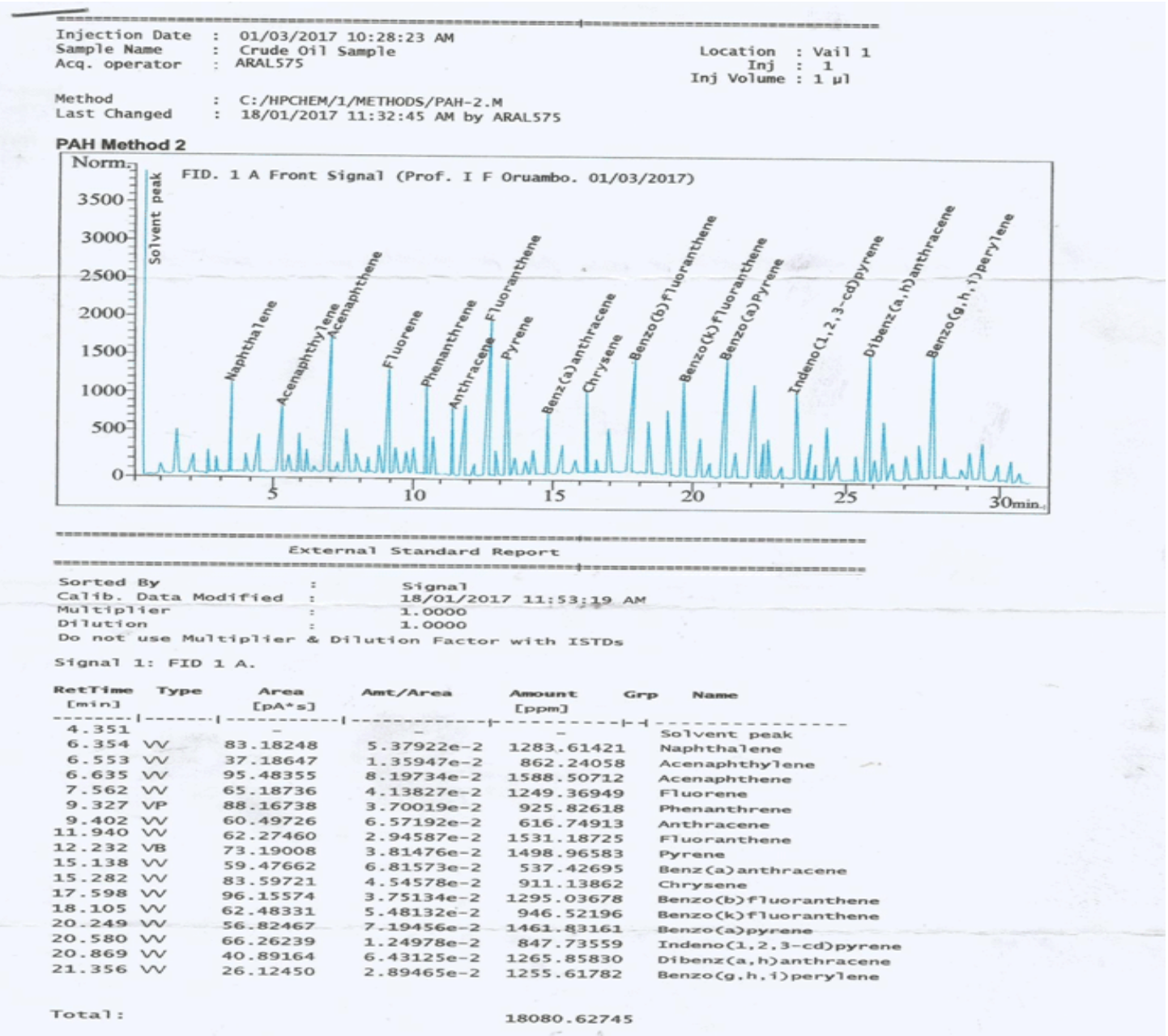

Figure 3: Chromatogram of PAHs in Non-exposed Bonny Light Crude Oil. 


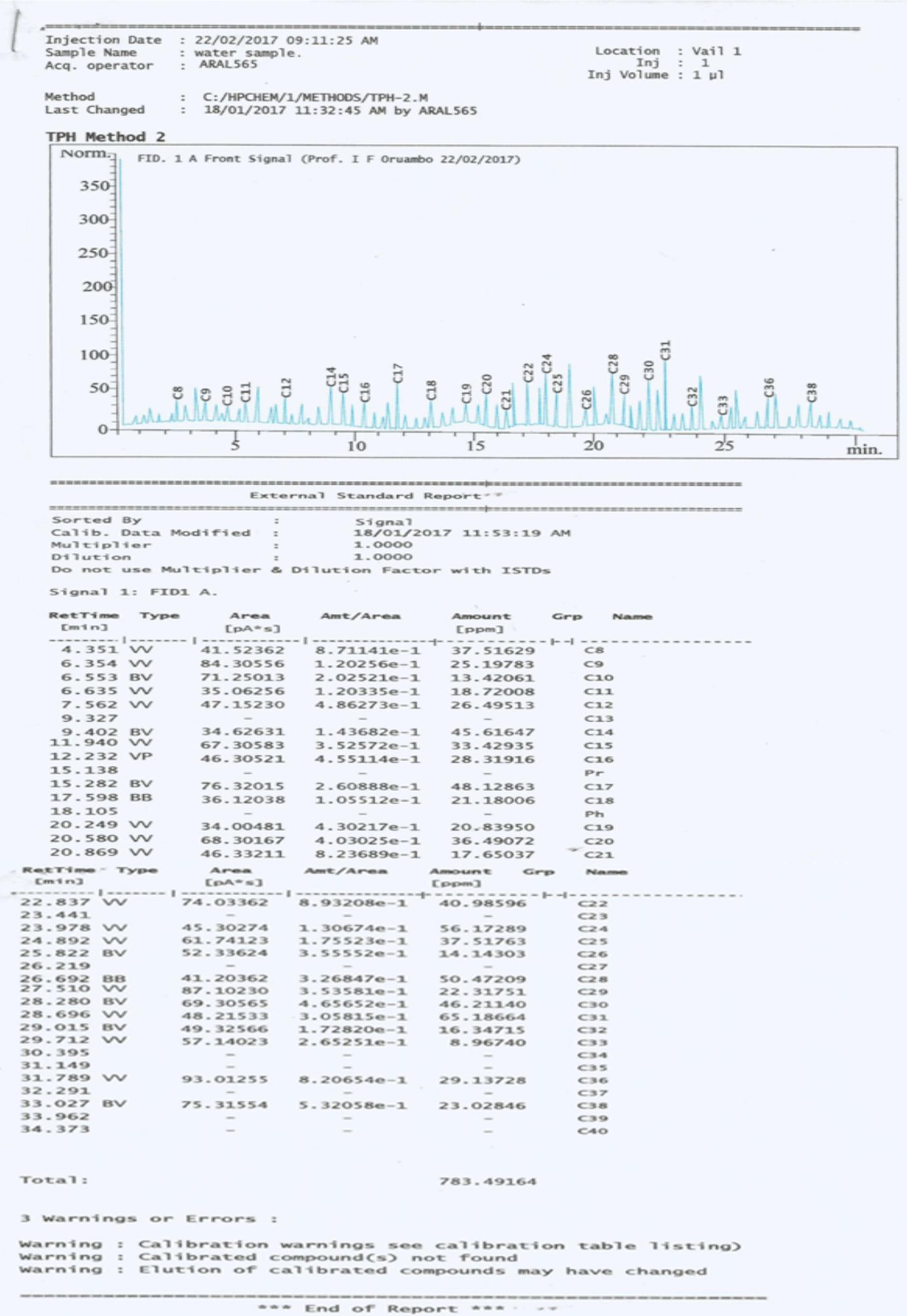

Figure 4: Chromatogram of TPH in atmospherically exposed water-underlying crude oil 
Table 1 shows the increasing peroxide values of fluorescent lighting-exposed virgin oil as a function of increasing duration [in hours], from zero hour [control] of $2 \mathrm{meq} / \mathrm{kg}$ to $56 \mathrm{meq} / \mathrm{kg}$ at 336hours of exposure. This result strongly suggests that the virgin oil was oxidized under prolonged exposure to fluorescentlighting.

Subsequent treatment of male albino rats to non-exposed VOO, or FPVOO, or APVOO, or PCO at two dose levels continuously for 14 days as previously described show four different levels of effect on liver and kidney function indicators [OFMs].

Table 1: Peroxide value of fluorescent lighting exposed virgin olive oi

\begin{tabular}{|c|c|}
\hline Hours & Peroxide Value (meq/kg) \\
\hline 0 & 2 \\
\hline 12 & 4 \\
\hline 24 & 6 \\
\hline 36 & 8 \\
\hline 48 & 10 \\
\hline 60 & 12 \\
\hline 72 & 14 \\
\hline 84 & 16 \\
\hline 96 & 18 \\
\hline 108 & 20 \\
\hline 120 & 22 \\
\hline 132 & 24 \\
\hline 144 & 26 \\
\hline 156 & 28 \\
\hline 168 & 30 \\
\hline 180 & 32 \\
\hline 192 & 34 \\
\hline 204 & 36 \\
\hline 216 & 38 \\
\hline 228 & 40 \\
\hline 240 & 42 \\
\hline 252 & 44 \\
\hline 264 & 46 \\
\hline 276 & 48 \\
\hline 288 & 50 \\
\hline 300 & 52 \\
\hline 312 & 54 \\
\hline 324 & 56 \\
\hline 336 & 56 \\
\hline
\end{tabular}

In Table 2 [i.e. liver function indices, LFI] values of alkaline phosphatase [ALP] and gamma glutamyl transferase [GGT] show the greatest increase over controls in the rats treated with FPVOO, but were decreased in PCO-treated rats. ALP is an essential liver function enzyme and thus this result may suggest risk to proper function in people who consume fluorescent-exposed olive oil as occurs in supermarket shelves. Vegetable oil that is sunlight exposed does not impair ALP as shown in Table 2. According to Shastry et.al, the high level of these enzymes suggest hepatic injury [9].

Rats treated with $4 \mathrm{ml}$ of FPVOO showed the least ALT increase over control as against those treated with $4 \mathrm{ml}$ of water underlying crude oil which showed $317 \%$ increase over control. Also, ALT increase over control was observed to follow a dose dependent pattern in rat treated with APVOO and PCO. This also implies that the higher the dose the greater the impact/effect on the organ which goes further to imply the higher the level of the enzymes in the blood. Similarly, in Table 2, ALP is increased sharply in rats treated with two doses of FPVOO but not by APVOO or PCO. This may be related to activation of hydrolytic enzymes which are designed to hydrolyze prodrugs [6]. Fluorescentinduced oxidation of the polyunsaturated hydrocarbons in virgin vegetable oil may have resulted in production of secondary metabolites that induce the observed changes in ALP levels.

Total bilirubin [TB] values are shown to be increased strongly over controls in rats treated with sunlight-exposed virgin oil [APVOO] and sunlight exposed water underlying crude oil [PCO]. These may suggest interference with bioactivation formation of bile and biliary secretion function of the liver, as abnormal bilirubin level is related to probable risk to jaundice in people who are chronically-exposed to oxidized vegetable oil and water underlying crude oil. Interestingly, atmospherically-exposed/ oxidized virgin oil and water underlying crude oil induced sharp increases in total bilirubin. This is not totally unusual because toxicities of endogenous substances are sometimes targetspecific which may be the case in these results.

Table 3 shows similar differentials in effects of FPVOO, APVOO, and PCO on selected kidney function indices. For example, APVOO and PCO induced sharp decreases of $\mathrm{Na}+$ and urea levels. Creatinine levels increased over control in a dose dependent manner in rats treated with water underlying crude oil.

In Table 3 [i.e. kidney function indices, KFI] values of sodium $\left[\mathrm{Na}^{+}\right]$, urea, bicarbonate and chloride show great increases over controls in the rats treated with FPVOO, but were decreased in APVOO-treated rats. Almost all the KFI values show increase over control in rats treated with PCO at the two dose levels except sodium and bicarbonate which was a bit lower than the control at $2 \mathrm{ml}$ dose level. This is a clear indication that kidney function was greatly impacted by these product of photo-oxidation.

\section{Conclusion}

The results of the study suggest that continuous exposure of vegetable oil [in transparent container] and water underlying crude oil to indoor fluorescent lighting or to atmospheric sunlight respectively result in the oxidation of the polycyclic aromatic hydrocarbons to potentially toxic secondary metabolites. 
Table 2: Liver function levels in serum of rats treated with fluorescent lighting exposed and sunlight exposed virgin oil and water underlying crude oil.

\begin{tabular}{|c|c|c|c|c|c|c|}
\hline Treatment & ALT (U/I) & $\operatorname{AST}(U / I)$ & $\operatorname{ALP}(U / I)$ & T.P (g/dl) & T.B (mmol/l) & GGT (U/I) \\
\hline Group A & $34.00 \pm 0.00$ & $36.00 \pm 0.00$ & $145.70 \pm 3.00$ & $16.60 \pm 0.36$ & $4.09 \pm 0.08$ & $97.65 \pm 1.30$ \\
\hline Group B & $43.33 \pm 4.51$ & $43.00 \pm 1.73$ & $156.67 \pm 3.89$ & $21.01 \pm 0.05$ & $4.12 \pm 0.05$ & $110.46 \pm 1.72$ \\
\hline Group C & $34.00 \pm 7.07$ & $31.50 \pm 6.36$ & $50.21 \pm 13.85$ & $23.66 \pm 1.48$ & $17.48 \pm 2.46$ & $35.58 \pm 2.28$ \\
\hline Group D & $50.5 \pm 11.33$ & $27.00 \pm 0.00$ & $35.70 \pm 0.00$ & $22.14 \pm 2.82$ & $18.01 \pm 0.46$ & $33.43 \pm 3.88$ \\
\hline Group E & $22.33 \pm 2.31$ & $30.00 \pm 0.00$ & $93.65 \pm 10.35$ & $17.82 \pm 1.12$ & $9.43 \pm 1.14$ & $77.20 \pm 8.14$ \\
\hline Group F & $37.33 \pm 2.89$ & $43.00 \pm 1.73$ & $136.61 \pm 6.47$ & $20.33 \pm 0.57$ & $5.98 \pm 0.89$ & $59.17 \pm 6.15$ \\
\hline Group G & $36.50 \pm 16.26$ & $27.00 \pm 0.00$ & $39.92 \pm 10.88$ & $22.42 \pm 1.34$ & $13.43 \pm 0.36$ & $52.35 \pm 2.73$ \\
\hline Group H & $71.00 \pm 32.53$ & $30.00 \pm 0.00$ & $34.46 \pm 3.15$ & $23.28 \pm 2.82$ & $19.33 \pm 1.42$ & $44.38 \pm 2.60$ \\
\hline Group I & $17.00 \pm 0.00$ & $17.00 \pm 1.73$ & $31.86 \pm 5.08$ & $10.48 \pm 0.20$ & $1.78 \pm 0.14$ & $32.44 \pm 3.22$ \\
\hline
\end{tabular}

Group A = FPVOO (2ml); Group B = FPVOO (4ml); Group C = APVOO (2ml); Group D = APVOO (4ml); Group E = Control VOO (2ml); Group F = Control VOO (4ml); Group G = PCO (2ml); Group H = PCO (4ml); Group I = External Control;

Table 3: Kidney function levels in serum of rats treated with fluorescent lighting exposed and sunlight exposed virgin oil and water underlying crude oil.

\begin{tabular}{|c|c|c|c|c|c|c|}
\hline Treatment & Na+(mmol/1) & K+(mmol/l) & Creatinine(mmol/l) & Urea(mmol/l) & Bicarbonate(mmol/l) & Chloride(mmol/l) \\
\hline Group A & $184.13 \pm 1.56$ & $3.15 \pm 0.08$ & $40.27 \pm 0.14$ & $20.09 \pm 0.31$ & $83.92 \pm 0.59$ \\
\hline Group B & $188.47 \pm 0.39$ & $4.19 \pm 1.01$ & $43.63 \pm 0.72$ & $23.78 \pm 0.76$ & $92.34 \pm 0.33$ \\
\hline Group C & $84.32 \pm 8.04$ & $5.84 \pm 1.19$ & $2.76 \pm 0.41$ & $8.51 \pm 0.11$ & $35.53 \pm 3.85$ \\
\hline Group D & $88.58 \pm 11.93$ & $6.25 \pm 0.78$ & $6.52 \pm 2.94$ & $9.60 \pm 1.02$ & $43.49 \pm 4.84$ \\
\hline Group E & $162.02 \pm 0.42$ & $3.67 \pm 0.40$ & $46.89 \pm 0.74$ & $12.02 \pm 0.76$ & $72.22 \pm 1.69$ \\
\hline Group F & $176.60 \pm 0.34$ & $4.24 \pm 0.05$ & $52.79 \pm 1.00$ & $16.72 \pm 0.52$ & $78.25 \pm 0.13$ \\
\hline Group G & $61.42 \pm 0.87$ & $6.25 \pm 0.21$ & $10.12 \pm 0.98$ & $8.72 \pm 0.92$ & $42.13 \pm 0.93$ \\
\hline Group H & $88.73 \pm 19.21$ & $6.45 \pm 0.64$ & $30.13 \pm 2.02$ & $9.41 \pm 0.18$ & $54.77 \pm 0.87$ \\
\hline Group I & $79.42 \pm 11.83$ & $5.18 \pm 0.11$ & $2.04 \pm 0.74$ & $8.33 \pm 0.19$ & $49.16 \pm 9.65$ \\
\hline
\end{tabular}

Group A = FPVOO (2ml); Group B = FPVOO (4ml); Group C = APVOO (2ml); Group D = APVOO (4ml); Group E = Control VOO (2ml); Group F = Control VOO (4ml); Group G = PCO (2ml); Group H = PCO (4ml); Group I = External Control;

In Table 2 and 3 the probable hepato-and nephro-toxicities by treatment of adult male albino rats with the secondary products of fluorescent-and sunlight induced photo-oxidation, as judged by effects to liver and kidney Function Indices, suggest that the liver may be more adversely affected than the kidney.

\section{Recommendations}

1. The obvious implication of these results is that vegetable oil should be stored in non-transparent/opaque bottles protected from fluorescent lighting while on supermarket shelves and in open general markets.

2. Commercial producers should be mandated by law/legislation to implement no 1 .

3. Crude oil spillages in the Nigerian Niger Delta region is now accepted to be a consequence to be endured. These results, however, suggest that proactive measures need to be taken to protect the vulnerable from undue exposure to the products of photo-oxidation of spilled crude oil.

\section{References}

1. Bach RD,Ayala PY, Schleger HB.A Reassessment of the Bond Dissociation Energies of Peroxides. J Amer Chem Soc. 1996;118(50):12758-12765.

2. Ehrdard $M$ and Wever RR. Formation of Low Molecular Weight Carbonyl Compounds by Sensitized Photo-chemical Decomposition of Aliphatic Hydrocarbons in Seawater. J Anal Chem. 1991;339(10):772779. Doi:10.1007/BF00321742

3. Harayanca S, Kishira H, Kassi Y, Shustsub K. Petroleum Biodegradation in Marine Environments. J. Molecules Microbiol. Biotechnol. 1999;1(1):63-70.

4. Hartmul Jaesclike. Toxic Responses of the liver, In: Casarett \& Doull's Toxicology, The Basic Science of Poisons, 8th Edition. 2013;

5. Igwe FU, Oruambo IF, Ekweozor IK, Oruamabo PP. Changes in the Blood Constituents of Adult Albino Rats following Dermal Exposure to Bonny Light Crude Oil. J Nigeria Enveron Soc. 2008;4(4):76-85.

6. Obediah GA and Oruambo IF. Blood Retention Pattern of Polycyclic Aromatic Hydrocarbons in Guinea-Pigs Treated with Crude Oil. International Journal of Science and Research (IJSR). 2016;5(4):361364 . 
7. Oruambo IF, Idabor L, Kachikwu S. Dose related Decrease in Activites of Liver Amino Transferases, and increases in RNA concentrations Following Treatment of Guinea-pigs with Bonny Light Crude Oil. Global J Environ Sci. 2008;7(1):9-12.

8. Potti S, Dondi D, Fagnoni M, Aibini A. Photo-Chemistry in Synthesis: Where, When and Why? Pure Applied Chemistry. 2007;79(11):19291939. Doi: 10.1351/pac200779111929
9. Shastry CS, Patel N Ambala, J Hand, Aswathranrayana BJ. Evaluation of effect of reused edible oil on vital organs of Wistar rats. Journal of Health Science. 2011;1(4)10-15.

10. Shrestha A and Kishen A. Polyatomic Chitosan-conjugated Photo Sensitizer for Antibacterial Photodynamic Therapy. Photo-chem Photobiol. 2012;88(3):577-583. Doi: 10.1111/j.1751-1097.2011.01026.x 